\title{
BMJ Open Tobacco and alcohol use among drug users receiving methadone maintenance treatment: a cross-sectional study in a rural prefecture of Yunnan Province, Southwest China
}

\author{
Song Duan, ${ }^{1}$ Ziyi Jin, ${ }^{2,3}$ Xing Liu, ${ }^{2}$ Yuecheng Yang, ${ }^{1}$ Runhua Ye, ${ }^{1}$ Renhai Tang, ${ }^{1}$ \\ Meiyang Gao, ${ }^{2}$ Yingying Ding, ${ }^{2} \mathrm{Na} \mathrm{He}$
}

To cite: Duan S, Jin Z, Liu X, et al. Tobacco and alcohol use among drug users receiving methadone maintenance treatment: a cross-sectional study in a rural prefecture of Yunnan Province, Southwest China. BMJ Open 2017;7:e014643. doi:10.1136/bmjopen-2016014643

- Prepublication history for this paper is available online. To view these files please visit the journal online (http://dx.doi.org/10.1136/ bmjopen-2016-014643).

$\mathrm{SD}, \mathrm{ZJ}$ and $\mathrm{XL}$ contributed equally.

Received 10 October 2016 Revised 25 January 2017 Accepted 23 February 2017

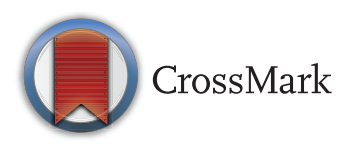

For numbered affiliations see end of article.

Correspondence to Dr Na He; nhe@fudan.edu.cn

\section{ABSTRACT}

Objective: To estimate the prevalence of ever, current and heavy tobacco and alcohol use and their correlates among patients undergoing methadone maintenance treatment (MMT).

Design: Cross-sectional study.

Setting: The study was conducted in all of the 5 MMT clinics in Dehong Prefecture, China.

Participants: 2121 (81.6\%) eligible MMT participants were included in the study population.

Analysis: Ordinal logistic regression was used to estimate the ORs and their 95\% Cls.

Results: The overall prevalence of ever, current and heavy smoking was $98.6 \%, 97.8 \%$ and $66.3 \%$, respectively; while that of ever, current and hazardous alcohol drinking was $86.6 \%, 58.6 \%$ and $16.6 \%$, respectively. Among HIV-infected participants, the proportions of those experiencing harmful effects of tobacco and alcohol on AIDS were $53.6 \%$ and $72.5 \%$, respectively, and $16.9 \%$ and $49.3 \%$ had ever tried to quit after diagnosis with HIV. After adjusting for potential confounders, heavier smokers and more hazardous drinkers were more likely to be men, older and less educated. Ethnic minorities were less likely to heavily smoke, but more likely to engage in hazardous drinking. In addition, hazardous drinking was negatively associated with longer years of MMT and HIV infection. Moreover, heavier smoking $\left(\mathrm{OR}_{\geq 2}=2.08\right.$, $95 \% \mathrm{Cl} 1.16$ to 3.73 ) and more hazardous drinking $\left(0 \mathrm{R}_{\geq 2}=2.46,95 \% \mathrm{Cl} 1.53\right.$ to 3.97$)$ were positively associated with having multiple sexual partners, and both were positively associated with each other.

Conclusions: The prevalence of tobacco and alcohol consumption was extraordinarily high among MMT participants in China, suggesting the urgent need of enhancing MMT patients' awareness of the harmful effects of tobacco and alcohol consumption and implementing comprehensive education and effective intervention programmes.

\section{INTRODUCTION}

China's methadone maintenance treatment (MMT) programme started with eight pilot
Strengths and limitations of this study

- This is the first study specifically examining tobacco and alcohol use and their correlates among drug users receiving methadone maintenance treatment (MMT), with a large sample size in China.

- The results provide a better understanding of the prevalence and severity of current tobacco and alcohol consumption among MMT patients, particularly among those infected with HIV, helping to target population at higher risk for tobacco/ alcohol-related diseases in the MMT population.

- This cross-sectional study is unable to make causal inferences between associated factors and tobacco and alcohol use.

- Self-reported data were used for risk behaviours, so information bias may exist.

- Participants were recruited from Dehong Prefecture; the findings might not be generalisable to MMT patients in other areas.

sites in five provinces in March 2004, scaled up nationwide in June 2006 and has become one of the largest opioid-substitution treatment and care systems in the world. ${ }^{12}$ By the end of 2012, a total of 756 community-based MMT clinics had been established in 28 provinces and had provided treatment for 384479 drug users (DUs). ${ }^{3}$ At the end of 2015, 167600 DUs were receiving treatment in 785 MMT clinics located in 29 provinces. Owing to successful implementation during the past decade, the MMT programme in China has drastically reduced drug use and related morbidity and mortality, improved quality of life as well as the social and family well-being of DUs. ${ }^{12}{ }^{4}$ As MMT patients live longer because of the effectiveness of MMT, unhealthy lifestyles such as smoking and alcohol drinking have been suggested to be major causes of excess mortality for DUs. ${ }^{5}$ 
Furthermore, more than $10 \%$ and $60 \%$ of MMT patients are living with HIV and HCV, respectively, and are therefore at even higher risk of onset and rapid progression of comorbidities associated with tobacco and alcohol use. $^{6-10}$

Tobacco and alcoholic beverages are highly addictive substances and widely consumed throughout the world, especially in China. ${ }^{11} 12$ Tobacco and alcohol consumption have serious effects on public health and are regarded as the most common modifiable and preventable risk factors for major non-communicable diseases. ${ }^{13}{ }^{14}$ They contain well-established group I carcinogens and are causally associated with development of certain cancers, ${ }^{15}$ and further contribute to the increasing health burden among persons living with HIV/AIDS (PLWHA) ${ }^{16}$ Globally, a much higher prevalence of smoking (79-93\%) has been reported in active DUs and MMT patients than in the general population, ${ }^{17-24}$ whereas the prevalence of alcohol drinking was reported to be varying from $13 \%$ to $49 \%$, depending on definitions of alcohol consumption and study area. $^{25-33}$

However, no study has been designed to specifically examine tobacco and alcohol use among DUs receiving MMT, particularly those living with HIV in China. To fill this gap, we conducted a cross-sectional study to specifically examine tobacco and alcohol use and their correlates, and further to explore by gender and by HIV infection status among a large sample of DUs receiving MMT in Dehong Prefecture of Yunnan Province at China's southwest border, where the first China's indigenous HIV outbreak was reported in 146 infected heroin users in 1989, and injection drug use had been the predominant mode of HIV transmission through the early 2000s and continues to be an important source of HIV infection. ${ }^{34} 35$

\section{METHODS}

\section{Study design, setting and participants}

Details of the study design have been described in a previous publication, which showed that the prevalence of illicit drug use was $10.4 \%, 12.9 \%$ and $9.2 \%$ for morphine, methamphetamine and both of them, respectively, in the study population. ${ }^{36}$ In brief, a cross-sectional survey was conducted from June to July in 2014 in Dehong Prefecture in Yunnan Province, China. The participants were identified from all of the five MMT clinics in Dehong Prefecture and were former opioid users, aged 20 years or older, had registered as local residents for at least 6 months where the clinic was located and were capable of completing civil liability (eg, of age and sound mind). ${ }^{12}$

\section{Data collection}

All participants had to read and sign the informed consent form prior to participating in the survey.
Trained public health professionals within the local clinics collected epidemiological data through faceto-face interviews using a structured questionnaire at a private location. The questionnaire had been tested in an early study. ${ }^{37} 38$ The epidemiological data included basic demographic characteristics (gender, age, ethnicity, marital status and level of education), tobacco smoking (age at starting smoking, type of cigarettes, smoking frequency, smoking intensity and number of cigarettes smoked per day (CPD) in the past month), alcohol drinking (age at starting drinking, types of alcoholic beverage, drinking frequency and quantity of drinking in the past month), tobacco smoking and alcohol drinking status during MMT, knowledge and quitting behaviour for HIV-infected individuals and sexual behaviours (number of sexual partners in the last year). Information on age at starting drug use, length of taking MMT, daily methadone dosage and HIV and HCV infection status were obtained from the MMT Data System.

\section{Measures}

Ever smokers were defined as having smoked at least 100 cigarettes in their lifetime, ${ }^{39}$ while ever alcohol drinkers were defined as having drunk at least once per month for more than 1 year. ${ }^{37}$ Current smokers and current drinkers were defined as those who smoke or drank in the month prior to the interview. Current smokers were further asked about cigarette type (manufactured only, hand-rolled only or combination of the two), smoking frequency (none, sometimes or daily) and number of CPD in the past month. We summed absolute numbers of both types of cigarette, then defined heavy smoking as smoking at least $20 \mathrm{CPD}$, moderate smoking as 10-19 CPD and light smoking as 0-9 CPD. ${ }^{39}$ As distilled spirits and beer were the mostly common consumed alcoholic beverage, alcohol type was classified into three categories in the analysis: distilled spirits only, beer only or combination of the two. Current drinkers were further asked about frequency of drinking (none, occasionally (1-3 times/month), often (1-3 times/week), almost daily (at least 4 times/week) or daily) and quantity of alcohol consumed in the past month. Weekly consumption of pure ethanol $(\mathrm{g} /$ week $)$ on average were calculated with the standard of ethanol content of $4 \%$ for beer and $40 \%$ for distilled spirits, and a conversion factor of $0.79 .{ }^{40}$ According to the US National Institute on Alcohol Abuse and Alcoholism (NIAAA) guidelines for physicians, the definition of standard drinks was $14 \mathrm{~g}$ of pure alcohol per drink. And we defined hazardous level as having more than 14 drinks/week (196 g of alcohol) for men and more than 7 drinks/week $(98 \mathrm{~g}$ ) for women, and moderate level as drinking ethanol below hazardous levels. ${ }^{41}$ The frequency of drinking was dichotomised into 'often or daily' versus 'none or occasionally' in the analysis.

\section{Statistical analysis}

SAS V.9.2 package (SAS Institute) was used to clean and analyse the data. The distribution of participants' 
sociodemographic characteristics, information on taking MMT, and tobacco and alcohol consumption by gender and by HIV infection status were described and compared using the $\chi^{2}$ test or Fisher's exact test, where appropriate. Univariate ordinal logistic regressions were used to estimate ORs and their $95 \%$ CIs to examine the associations between smoking/drinking behaviours and related factors. In multiple regression, ordinal logistic regression models were performed with 'forced entry' of all variables selected based on prior knowledge and confounding assessment, for intensity of smoking and levels of alcohol drinking in the past month, respectively. These included gender (women/men), age (years, 1829/30-39/40-49/50-79), ethnicity (Han/Jingpo/Dai/ others), marital status (never married/in marriage/ divorced or widowed), education level (illiteracy or primary/middle/high or above), age at first drug use (years, $<25 / \geq 25$ ), length of MMT (years, $<1 / 1-5 />5$ ), daily methadone dose (mL, $\leq 30 / 31-60 / 61-99 / \geq 100)$, number of sexual partners in the last year $(0 / 1 / \geq 2)$, HIV infection(no/yes), HCV infection(no/yes), alcohol use in the past month (none/moderate/hazardous, except for alcohol use) and smoking intensity in the past month (low/moderate/high, except for smoking).

\section{RESULTS}

\section{Sociodemographic characteristics and status of HIV/HCV} infection

Characteristics of MMT participants overall and by gender are summarised in table 1. During the study period, a total of 2600 DUs were receiving MMT. However, 269 participants could not complete the questionnaire due to intoxication, disability (eg, deaf-mute) or severe mental disorders. These conditions were determined by clinical judgements of the trained public health professionals conducting the face-to-face interviews. In addition, 197 did not appear at MMT sites and 13 participants were excluded because of excessive missing data. Finally, 2121 (81.6\%) eligible MMT participants were included in the final analysis. Of the 2121 participants, $2041(96.2 \%)$ were men; the mean age was 40.8 years $(\mathrm{SD}=10.0)$. Jingpo $(42.9 \%)$ and Han $(41.7 \%)$ were the largest two ethnic groups. Seventy per cent of the participants were married, and $1063(50.1 \%)$ were illiterate or only had a primary school education. For HIV/HCV infection status, 451 (21.3\%) had western blot confirmed HIV infection, while $786(37.1 \%)$ were positive for serum HCV antibody. Male and female participants were significantly different in age, ethnicity and level of education.

\section{Drug use, MMT and sexual behaviour}

Among the study participants, 1199 (56.5\%) started using heroin before they were 25 years. System data showed that $1754(82.7 \%)$ had received MMT for more than 1 year and $669(31.5 \%)$ for more than 5 years and $411(20.2 \%)$ had daily methadone dosage $>100 \mathrm{~mL}$. For sexual behaviour, $75(3.5 \%)$ reported having had 2 or more sex partners in the last year. No statistically significant difference was observed between men and women in these behaviours.

\section{Tobacco use}

The overall characteristics of tobacco smoking among MMT participants are presented in table 2, and were compared by gender and further by HIV infection status. Among all participants, 2091 (98.6\%) were ever smokers, $2075(97.8 \%)$ self-reported as current smokers and $1276(61.0 \%)$ started smoking before they were 18 years; $1313(62.8 \%)$ had smoked for more than 20 years and $2079(98.0 \%)$ still smoked during MMT. Among current smokers, 1406 (66.3\%) smoked more than 20 cigarettes/day and were defined as heavy smokers in this study. Almost all smokers preferred manufactured cigarettes. Smoking patterns were all significantly different by gender, since men were more likely to smoke $(99.2 \%$ vs $83.8 \%, \mathrm{p}<0.001)$, were more likely to start smoking before the age of $18(61.8 \%$ vs $38.8 \%$, $\mathrm{p}<0.001$ ), had a longer duration of smoking (proportion of those who smoked for more than 20 years: $64.2 \%$ vs $20.9 \%, \mathrm{p}<0.001)$, had a higher frequency of smoking (proportion of those smoking daily: $93.4 \%$ vs $66.3 \%$, $\mathrm{p}<0.001$ ) and higher intensity (heavy smoking: $68.1 \%$ vs $20.0 \%, \mathrm{p}<0.001)$. When stratified by gender, HIV-positive individuals were more likely to start smoking before 18 in men $(74.1 \%$ vs $58.4 \%, \mathrm{p}<0.001)$ and women $(63.2 \%$ vs $29.2 \%, \mathrm{p}=0.010)$, and to prefer manufactured cigarettes $(95.8 \%$ vs $86.8 \%, \mathrm{p}<0.001)$ compared with HIVnegative participants in men. Among the HIV-infected individuals, $231(53.6 \%)$ thought smoking has harmful effect on AIDS disease progression, while only 73 (16.9\%) ever tried to quit smoking after being diagnosed with HIV, with no statistically significant difference by gender.

The prevalence of light, moderate and heavy smoking and associated factors of tobacco smoking intensity among MMT patients are presented and examined by ordinal logistic regression models in table 3. After adjusting for potential confounding factors, heavier smoking in the preceding month was positively associated with men (OR=13.10, 95\% CI 8.22 to 20.88), having more sexual partners in the past month $\left(\mathrm{OR}_{\geq 2}=2.08,95 \%\right.$ CI 1.16 to 3.73 ) and engaging in hazardous drinking $(\mathrm{OR}=1.57,95 \% \mathrm{CI} 1.18$ to 2.10$)$, and negatively associated with being an ethnic minority $\left(\mathrm{OR}_{\mathrm{Jingpo}}=0.63\right.$, $95 \%$ CI 0.51 to 0.79 ) and having received higher education $\left(\mathrm{OR}_{\text {high }}\right.$ or above $=0.72,95 \%$ CI 0.53 to 1.00$)$. Smoking intensity was not significantly associated with age, marital status, age at starting drug use, duration of taking MMT, daily methadone dose, or HIV or HCV infection.

\section{Alcohol use}

The characteristics of alcohol drinking among MMT participants are shown in table 4. Of all participants, 
Table 1 Characteristics of study participants

\begin{tabular}{|c|c|c|c|}
\hline Variables* & Total $(\mathrm{N}=\mathbf{2 1 2 1})$ & Male $(\mathrm{N}=2041)$ & Female $(\mathrm{N}=80)$ \\
\hline \multicolumn{4}{|l|}{ Age (years) $(p=0.024)$} \\
\hline $18-29$ & $251(11.8)$ & $243(11.9)$ & $8(10.0)$ \\
\hline $30-39$ & $811(38.2)$ & 769 (37.7) & $42(52.5)$ \\
\hline $40-49$ & $653(30.8)$ & 639 (31.3) & $14(17.5)$ \\
\hline $50-79$ & $406(19.1)$ & $390(19.1)$ & $16(20.0)$ \\
\hline \multicolumn{4}{|l|}{ Ethnicity $(p=0.023)$} \\
\hline Han & $885(41.7)$ & $854(41.8)$ & $31(38.8)$ \\
\hline Jingpo & 909 (42.9) & $880(43.1)$ & 29 (36.3) \\
\hline Dai & $249(11.7)$ & $231(11.3)$ & $18(22.5)$ \\
\hline Others & $78(3.7)$ & $76(3.7)$ & $2(2.5)$ \\
\hline \multicolumn{4}{|l|}{ Marital status $(p=0.156)$} \\
\hline Never married & $378(17.8)$ & $368(18.0)$ & $10(12.5)$ \\
\hline Current married & $1495(70.5)$ & $1439(70.5)$ & $56(70.0)$ \\
\hline Divorced or widowed & $248(11.7)$ & $234(11.5)$ & $14(17.5)$ \\
\hline \multicolumn{4}{|l|}{ Education level $(p=0.001)$} \\
\hline Illiteracy or primary & $1063(50.1)$ & $1038(50.9)$ & $25(31.3)$ \\
\hline Middle & $816(38.5)$ & 777 (38.1) & $39(48.8)$ \\
\hline High or above & $242(11.4)$ & $226(11.1)$ & $16(20.0)$ \\
\hline \multicolumn{4}{|c|}{ Age at first drug use (years) $(p=0.778)$} \\
\hline$<25$ & 1199 (56.5) & 1155 (56.6) & $44(55.0)$ \\
\hline$\geq 25$ & $922(43.5)$ & $886(43.4)$ & $36(45.0)$ \\
\hline \multicolumn{4}{|c|}{ Length of MMT (years) $(p=0.086)$} \\
\hline$<1$ & 367 (17.3) & $360(17.6)$ & $7(8.8)$ \\
\hline $1-5$ & $1085(51.2)$ & $1043(51.1)$ & $42(52.5)$ \\
\hline$>5$ & 669 (31.5) & 638 (31.3) & $31(38.8)$ \\
\hline \multicolumn{4}{|c|}{ Daily methadone dose $(\mathrm{mL})(\mathrm{p}=0.444)$} \\
\hline$\leq 30$ & $335(16.4)$ & $327(16.7)$ & $8(10.1)$ \\
\hline $31-60$ & $765(37.5)$ & 732 (37.3) & $33(41.8)$ \\
\hline $61-99$ & $528(25.9)$ & 508 (25.9) & $20(25.3)$ \\
\hline$\geq 100$ & $411(20.2)$ & $393(20.1)$ & $18(22.8)$ \\
\hline \multicolumn{4}{|c|}{ Number of sexual partners in the past year $(p=0.357)$} \\
\hline 0 & $601(28.4)$ & $581(28.5)$ & $20(25.0)$ \\
\hline 1 & $1441(68.1)$ & $1386(68.0)$ & $55(68.8)$ \\
\hline$\geq 2$ & $75(3.5)$ & $70(3.4)$ & $5(6.3)$ \\
\hline \multicolumn{4}{|l|}{ HIV infection $(p=0.405)$} \\
\hline No & $1670(78.7)$ & $1610(78.9)$ & $60(75.0)$ \\
\hline Yes & $451(21.3)$ & $431(21.1)$ & $20(25.0)$ \\
\hline \multicolumn{4}{|l|}{ HCV infection ( $p=0.698)$} \\
\hline No & $1335(62.9)$ & $1283(62.9)$ & $52(65.0)$ \\
\hline Yes & $786(37.1)$ & $758(37.1)$ & $28(35.0)$ \\
\hline
\end{tabular}

$1837(86.6 \%)$ were ever drinkers, $953(51.9 \%)$ started drinking before they were 18 years old, 1092 (59.4\%) had consumed alcohol for more than 20 years and 1555 $(73.3 \%)$ were still drinking during MMT and also in the past month prior to interview. The prevalence of current drinking, drinking often or daily and drinking at hazardous level were $58.6 \%, 24.5 \%$ and $16.6 \%$, respectively. Men had significantly different alcohol drinking patterns than women. Men were more likely to drink, had a longer duration of drinking, drank more distilled spirits, drank more and had a higher drinking frequency. In men, compared with HIV-uninfected individuals, HIV-infected cases had a lower prevalence of drinking during MMT (66.8\% vs $76.5 \%, \mathrm{p}<0.001)$, smaller frequency of drinking in the past month (proportion of drinking often and daily: $17.6 \%$ vs $27.3 \%, \mathrm{p}<0.001)$, less distilled spirits use $(68.7 \%$ vs $72.5 \%, \mathrm{p}<0.001)$ and fewer had a hazardous level of drinking $(9.3 \%$ vs $19.1 \%, \mathrm{p}<0.001)$. No significant difference was seen in women when comparing by HIV status. Among those HIV cases, $266(72.5 \%)$ thought alcohol was harmful for AIDS disease progression, and $181(49.3 \%)$ had ever tried to quit drinking after diagnosed with HIV, with no significant difference between men and women.

The prevalence of none, moderate and hazardous alcohol drinking and associated factors among MMT patients were examined and shown in table 5. After adjusting for potential confounding factors, higher levels of alcohol drinking in the preceding month were 
Table 2 Characteristics of tobacco smoking among study participants $\dagger$

\begin{tabular}{|c|c|c|c|c|c|c|c|}
\hline \multirow[b]{2}{*}{ Variables } & \multirow[b]{2}{*}{ Total $(\mathrm{N}=2121)$} & \multirow[b]{2}{*}{ Male $(\mathrm{N}=2041)$} & \multirow[b]{2}{*}{ Female $(\mathrm{N}=80)$} & \multicolumn{2}{|l|}{ Male } & \multicolumn{2}{|l|}{ Female } \\
\hline & & & & $\begin{array}{l}\mathrm{HIV+} \\
(\mathrm{N}=431)\end{array}$ & $\begin{array}{l}\text { HIV- } \\
(\mathrm{N}=1610)\end{array}$ & $\begin{array}{l}\text { HIV+ } \\
(\mathrm{N}=20)\end{array}$ & $\begin{array}{l}\text { HIV- } \\
(\mathrm{N}=60)\end{array}$ \\
\hline Ever smoking & & $* * *$ & & & & & \\
\hline No & $30(1.4)$ & $17(0.8)$ & $13(16.3)$ & $2(0.5)$ & $15(0.9)$ & $1(5.0)$ & $12(20.0)$ \\
\hline Yes & 2091 (98.6) & 2024 (99.2) & 67 (83.8) & $429(99.5)$ & $1595(99.1)$ & $19(95.0)$ & $48(80.0)$ \\
\hline \multicolumn{2}{|c|}{ Smoking initiation age (years) } & $* * \star$ & & $\star * *$ & & * & \\
\hline$\geq 18$ & $815(39.0)$ & $774(38.2)$ & $41(61.2)$ & $111(25.9)$ & $663(41.6)$ & $7(36.8)$ & $34(70.8)$ \\
\hline$<18$ & $1276(61.0)$ & $1250(61.8)$ & $26(38.8)$ & $318(74.1)$ & 932 (58.4) & $12(63.2)$ & $14(29.2)$ \\
\hline \multicolumn{2}{|l|}{ Smoking years } & 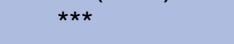 & & & & & \\
\hline$<20$ & $778(37.2)$ & $725(35.8)$ & $53(79.1)$ & $149(34.7)$ & $576(36.1)$ & $13(68.4)$ & $40(83.3)$ \\
\hline$\geq 20$ & $1313(62.8)$ & 1299 (64.2) & $14(20.9)$ & $280(65.3)$ & $1019(63.9)$ & $6(31.6)$ & $8(16.7)$ \\
\hline \multicolumn{2}{|l|}{ Smoking during MMT } & $* \star \star *$ & & & & & \\
\hline No & $42(2.0)$ & $22(1.1)$ & $20(25.0)$ & $4(0.9)$ & $18(1.1)$ & $2(10.0)$ & $18(30.0)$ \\
\hline Yes & 2079 (98.0) & 2019 (98.9) & $60(75.0)$ & $427(99.1)$ & $1592(98.9)$ & $18(90.0)$ & $42(70.0)$ \\
\hline \multicolumn{2}{|c|}{ Smoking frequency in the past month } & 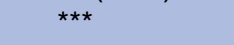 & & & & & \\
\hline None & $46(2.2)$ & $25(1.2)$ & $21(26.3)$ & $3(0.7)$ & $22(1.4)$ & $2(10.0)$ & $19(31.7)$ \\
\hline Sometimes & $116(5.5)$ & $110(5.4)$ & $6(7.5)$ & $15(3.5)$ & $95(5.9)$ & $3(15.0)$ & $3(5.0)$ \\
\hline Daily & 1959 (92.4) & 1906 (93.4) & $53(66.3)$ & $413(95.8)$ & $1493(92.7)$ & $15(75.0)$ & $38(63.3)$ \\
\hline \multicolumn{2}{|l|}{ Smoking types } & 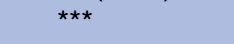 & & $\star \star \star *$ & & & \\
\hline Manufactured only & 1845 (88.9) & $1788(88.7)$ & $57(96.6)$ & $410(95.8)$ & $1378(86.8)$ & $18(100.0)$ & $39(95.1)$ \\
\hline Hand-rolled only & $43(2.1)$ & $42(2.1)$ & $1(1.7)$ & $4(0.9)$ & $38(2.4)$ & $0(0.0)$ & $1(2.4)$ \\
\hline Both above & $187(9.0)$ & $186(9.2)$ & $1(1.7)$ & $14(3.3)$ & $172(10.8)$ & $0(0.0)$ & $1(2.4)$ \\
\hline \multicolumn{2}{|c|}{ Smoking intensity in the past month } & 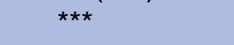 & & & & & \\
\hline Low & $226(10.7)$ & $177(8.7)$ & $49(61.3)$ & $42(9.7)$ & $135(8.4)$ & $11(55.0)$ & $38(63.3)$ \\
\hline Moderate & $489(23.1)$ & $474(23.2)$ & $15(18.8)$ & 96 (22.3) & 378 (23.5) & $4(20.0)$ & $11(18.3)$ \\
\hline Heavy & $1406(66.3)$ & $1390(68.1)$ & $16(20.0)$ & $293(68.0)$ & $1097(68.1)$ & $5(25.0)$ & $11(18.3)$ \\
\hline \multicolumn{8}{|c|}{ Attitude to effect of smoking on AIDS } \\
\hline Good & $6(1.4)$ & $6(1.5)$ & $0(0.0)$ & $6(1.5)$ & - & $0(0.0)$ & - \\
\hline Harmful & $231(53.6)$ & $219(53.0)$ & $12(66.7)$ & $219(53.0)$ & - & $12(66.7)$ & - \\
\hline No effect & $194(45.0)$ & $188(45.5)$ & $6(33.3)$ & $188(45.5)$ & - & $6(33.3)$ & - \\
\hline \multicolumn{8}{|c|}{ Ever tried to quit smoking after diagnosed with HIV } \\
\hline Yes & 73 (16.9) & $69(16.7)$ & $4(22.2)$ & $69(16.7)$ & - & $4(22.2)$ & - \\
\hline No & $358(83.1)$ & $344(83.3)$ & $14(77.8)$ & $344(83.3)$ & - & $14(77.8)$ & - \\
\hline
\end{tabular}


Table 3 Prevalence and associated factors of tobacco smoking intensity among study participants

\begin{tabular}{|c|c|c|c|c|c|}
\hline Variables & $\begin{array}{l}\text { Low } \\
(\mathrm{N}=226)\end{array}$ & $\begin{array}{l}\text { Moderate } \\
(\mathrm{N}=489)\end{array}$ & $\begin{array}{l}\text { High } \\
(\mathrm{N}=1406)\end{array}$ & Crude OR $(95 \% \mathrm{Cl})$ & Adjusted OR (95\% Cl)† \\
\hline \multicolumn{6}{|l|}{ Gender } \\
\hline Female & 49 (61.3) & $15(18.8)$ & $16(20.0)$ & 1.00 & 1.00 \\
\hline Male & $177(8.7)$ & 474 (23.2) & $1390(68.1)$ & $14.04(8.96 \text { to } 22.01)^{\star \star \star}$ & $13.10(8.22 \text { to } 20.88)^{\star \star \star}$ \\
\hline \multicolumn{6}{|l|}{ Age (years) } \\
\hline $18-29$ & $33(13.1)$ & $61(24.3)$ & $157(62.5)$ & 1.00 & 1.00 \\
\hline 30-39 & 89 (11.0) & 197 (24.3) & $525(64.7)$ & $1.12(0.84$ to 1.49$)$ & $1.20(0.88$ to 1.64$)$ \\
\hline $40-49$ & $62(9.5)$ & $142(21.7)$ & 449 (68.8) & $1.34(0.99$ to 1.80$)$ & $1.30(0.92$ to 1.84$)$ \\
\hline $50-79$ & $42(10.3)$ & 89 (21.9) & $275(67.7)$ & $1.27(0.92$ to 1.75$)$ & $1.34(0.90$ to 2.00$)$ \\
\hline \multicolumn{6}{|l|}{ Ethnicity } \\
\hline Han & $79(8.9)$ & $194(21.9)$ & 612 (69.2) & 1.00 & 1.00 \\
\hline Jingpo & $106(11.7)$ & $224(24.6)$ & $579(63.7)$ & $0.78(0.64 \text { to } 0.94)^{*}$ & $0.63(0.51 \text { to } 0.79)^{\star \star \star}$ \\
\hline Dai & $28(11.2)$ & $56(22.5)$ & $165(66.3)$ & $0.86(0.64$ to 1.16$)$ & $0.77(0.56$ to 1.06$)$ \\
\hline Others & $13(16.7)$ & $15(19.2)$ & $50(64.1)$ & $0.73(0.46$ to 1.17$)$ & 0.64 (0.39 to 1.03$)$ \\
\hline \multicolumn{6}{|l|}{ Marital status } \\
\hline Never married & $46(12.2)$ & $97(25.7)$ & 235 (62.2) & 1.00 & 1.00 \\
\hline Current married & $152(10.2)$ & $336(22.5)$ & $1007(67.4)$ & 1.25 (0.99 to 1.57$)$ & $1.18(0.87$ to 1.60$)$ \\
\hline Divorced or widowed & $28(11.3)$ & $56(22.6)$ & $164(66.1)$ & $1.17(0.84$ to 1.63$)$ & $1.21(0.85$ to 1.72$)$ \\
\hline \multicolumn{6}{|l|}{ Education level } \\
\hline Illiteracy or primary & $112(10.5)$ & $221(20.8)$ & $730(68.7)$ & 1.00 & 1.00 \\
\hline Middle & $81(9.9)$ & $207(25.4)$ & $528(64.7)$ & $0.86(0.71$ to 1.05$)$ & $0.86(0.69$ to 1.07$)$ \\
\hline High or above & $33(13.6)$ & $61(25.2)$ & $148(61.2)$ & $0.72(0.54 \text { to } 0.95)^{*}$ & $0.72(0.53 \text { to } 1.00)^{*}$ \\
\hline \multicolumn{6}{|c|}{ Age at first drug use (years) } \\
\hline$<25$ & $122(10.2)$ & $285(23.8)$ & $792(66.1)$ & 1.00 & 1.00 \\
\hline$\geq 25$ & $104(11.3)$ & $204(22.1)$ & $614(66.6)$ & $1.00(0.84$ to 1.20$)$ & $0.90(0.72$ to 1.13$)$ \\
\hline \multicolumn{6}{|l|}{ Length of MMT (years) } \\
\hline$<1$ & $32(8.7)$ & $86(23.4)$ & $249(67.8)$ & 1.00 & 1.00 \\
\hline $1-5$ & $114(10.5)$ & $240(22.1)$ & $731(67.4)$ & $0.96(0.75$ to 1.23$)$ & $0.97(0.75$ to 1.26$)$ \\
\hline$>5$ & $80(12.0)$ & $163(24.4)$ & $426(63.7)$ & $0.81(0.62$ to 1.06$)$ & 0.81 (0.61 to 1.09$)$ \\
\hline \multicolumn{6}{|c|}{ Daily methadone dose $(\mathrm{mL})$} \\
\hline$\leq 30$ & $36(10.7)$ & $78(23.3)$ & $221(66.0)$ & 1.00 & 1.00 \\
\hline $31-60$ & $85(11.1)$ & $182(23.8)$ & $498(65.1)$ & $1.02(0.80$ to 1.30$)$ & $1.02(0.80$ to 1.32$)$ \\
\hline $61-99$ & $50(9.5)$ & $115(21.8)$ & $363(68.8)$ & $1.20(0.92$ to 1.57$)$ & $1.29(0.97$ to 1.70$)$ \\
\hline$\geq 100$ & $51(12.4)$ & $82(20.0)$ & $278(67.6)$ & $1.10(0.83$ to 1.46$)$ & $1.25(0.92$ to 1.70$)$ \\
\hline \multicolumn{6}{|c|}{ Number of sexual partners in the last year } \\
\hline 0 & $76(12.6)$ & $140(23.3)$ & $385(64.1)$ & 1.00 & 1.00 \\
\hline 1 & $143(9.9)$ & $337(23.4)$ & $961(66.7)$ & $1.31(1.08 \text { to } 1.58)^{\star *}$ & $1.15(0.88$ to 1.51$)$ \\
\hline$\geq 2$ & $7(9.3)$ & $11(14.7)$ & $57(76.0)$ & $1.55(1.27 \text { to } 1.89)^{\star \star *}$ & $2.08(1.16 \text { to } 3.73)^{*}$ \\
\hline \multicolumn{6}{|c|}{ Alcohol use in the past month } \\
\hline None & $129(14.7)$ & $203(23.1)$ & $547(62.2)$ & 1.00 & 1.00 \\
\hline Moderate & $63(7.1)$ & $227(25.5)$ & $599(67.4)$ & $1.36(1.12 \text { to } 1.64)^{\star *}$ & $1.19(0.97$ to 1.47$)$ \\
\hline Hazardous & $34(9.6)$ & 59 (16.7) & 260 (73.7) & $1.71(1.31 \text { to } 2.24)^{\star \star \star}$ & $1.57(1.18 \text { to } 2.10)^{\star \star *}$ \\
\hline \multicolumn{6}{|l|}{ HIV infection } \\
\hline No & $173(10.4)$ & 389 (23.3) & 1108 (66.3) & 1.00 & 1.00 \\
\hline Yes & $53(11.8)$ & $100(22.2)$ & $298(66.1)$ & $0.97(0.78$ to 1.20$)$ & 1.00 (0.77 to 1.29$)$ \\
\hline \multicolumn{6}{|l|}{ HCV infection } \\
\hline No & $141(10.6)$ & $309(23.1)$ & 885 (66.3) & 1.00 & 1.00 \\
\hline Yes & $85(10.8)$ & $180(22.9)$ & 521 (66.3) & $1.00(0.83$ to 1.20$)$ & 0.99 (0.81 to 1.22$)$ \\
\hline
\end{tabular}

${ }^{*} p<0.05 ;{ }^{* *} p<0.01 ;{ }^{* * *} p<0.001$.

†Adjusted for potential confounding variables listed in the table by multivariate ordinal logistic regression analysis.

positively associated with being a man $(\mathrm{OR}=7.20,95 \%$ CI 3.83 to 13.54$)$, at an older age $\left(\mathrm{OR}_{40-49}=1.70,95 \% \mathrm{CI}\right.$ 1.23 to 2.35 ; $\mathrm{OR}_{50-79}=1.76$, $95 \%$ CI 1.22 to 2.53 ), being an ethnic minority $\left(\mathrm{OR}_{\mathrm{Jingpo}}=2.58,95 \%\right.$ CI 2.11 to 3.16 ; $\mathrm{OR}_{\text {Dai }}=3.08,95 \%$ CI 2.31 to 4.11 ), having two or more sexual partners in the last year $\left(\mathrm{OR}_{\geq 2}=2.46,95 \%\right.$ CI 1.53 to 3.97$)$ and being a heavy smoker $\left(\mathrm{OR}_{\mathrm{high}}=1.39,95 \%\right.$
CI 1.03 to 1.88 ). Heavier drinking was negatively associated with having received higher education $\left(\mathrm{OR}_{\text {middle }}=0.76,95 \%\right.$ CI 0.63 to 0.93 ; $\mathrm{OR}_{\text {high or above- }}$ $=0.65,95 \%$ CI 0.48 to 0.89 ), having a longer duration of MMT ( $\mathrm{OR}=0.77,95 \%$ CI 0.59 to 1.00$)$ and being HIV infected (OR=0.53, 95\% CI 0.42 to 0.68$)$. Quantity of alcohol consumption was not significantly associated 
Table 4 Characteristics of alcohol drinking among study participants

\begin{tabular}{|c|c|c|c|c|c|c|c|}
\hline \multirow[b]{2}{*}{ Variables } & \multirow[b]{2}{*}{ Total $(\mathrm{N}=\mathbf{2 1 2 1})$} & \multirow[b]{2}{*}{ Male $(\mathrm{N}=2041)$} & \multirow[b]{2}{*}{ Female $(\mathrm{N}=\mathbf{8 0})$} & \multicolumn{2}{|l|}{ Male } & \multicolumn{2}{|l|}{ Female } \\
\hline & & & & $\begin{array}{l}\text { HIV+ } \\
(\mathrm{N}=431)\end{array}$ & $\begin{array}{l}\text { HIV- } \\
(N=1610)\end{array}$ & $\begin{array}{l}\mathrm{HIV+} \\
(\mathrm{N}=20)\end{array}$ & $\begin{array}{l}\text { HIV- } \\
(\mathrm{N}=60)\end{array}$ \\
\hline Ever alcohol drinking & & *** & & & & & \\
\hline No & $284(13.4)$ & $257(12.6)$ & $27(33.8)$ & 60 (13.9) & $197(12.2)$ & $6(30.0)$ & $21(35.0)$ \\
\hline Yes & $1837(86.6)$ & $1784(87.4)$ & $53(66.3)$ & $371(86.1)$ & $1413(87.8)$ & $14(70.0)$ & $39(65.0)$ \\
\hline \multicolumn{8}{|c|}{ Drinking initiation age (years) } \\
\hline$\geq 18$ & $884(48.1)$ & $854(47.9)$ & $30(56.6)$ & $149(40.2)$ & $705(49.9)$ & $5(35.7)$ & $25(64.1)$ \\
\hline$<18$ & $953(51.9)$ & $930(52.1)$ & $23(43.4)$ & $222(59.8)$ & $708(50.1)$ & 9 (64.3) & $14(35.9)$ \\
\hline \multicolumn{8}{|l|}{ Drinking years } \\
\hline$<20$ & $745(40.6)$ & $708(39.7)$ & $37(69.8)$ & $155(41.8)$ & $553(39.1)$ & $11(78.6)$ & $26(66.7)$ \\
\hline$\geq 20$ & $1092(59.4)$ & $1076(60.3)$ & $16(30.2)$ & $216(58.2)$ & $860(60.9)$ & $3(21.4)$ & $13(33.3)$ \\
\hline \multicolumn{8}{|l|}{ Drinking during MMT } \\
\hline No & $566(26.7)$ & $521(25.5)$ & $45(56.3)$ & $143(33.2)$ & $378(23.5)$ & $10(50.0)$ & $35(58.3)$ \\
\hline Yes & 1555 (73.3) & $1520(74.5)$ & $35(43.8)$ & $288(66.8)$ & $1232(76.5)$ & $10(50.0)$ & $25(41.7)$ \\
\hline \multicolumn{8}{|c|}{ Drinking frequency in the past month } \\
\hline None and occasionally & $1602(75.5)$ & $1526(74.8)$ & $76(95.0)$ & $355(82.4)$ & $1171(72.7)$ & $19(95.0)$ & $57(95.0)$ \\
\hline Often and daily & $519(24.5)$ & $515(25.2)$ & $4(5.0)$ & 76 (17.6) & $439(27.3)$ & $1(5.0)$ & $3(5.0)$ \\
\hline \multicolumn{8}{|l|}{ Drinking types } \\
\hline Distilled spirits only & $563(45.3)$ & $560(45.6)$ & $3(23.1)$ & $90(44.8)$ & $470(45.7)$ & $0(0.0)$ & $3(30.0)$ \\
\hline Beer only & $355(28.6)$ & 346 (28.2) & 9 (69.2) & $63(31.3)$ & $283(27.5)$ & $2(66.7)$ & $7(70.0)$ \\
\hline Both of the above & $324(26.1)$ & $323(26.3)$ & $1(7.7)$ & $48(23.9)$ & $275(26.8)$ & 1 (33.3) & $0(0.0)$ \\
\hline \multicolumn{8}{|c|}{ Alcohol use in the past month } \\
\hline None & $879(41.4)$ & $812(39.8)$ & $67(83.8)$ & $230(53.4)$ & $582(36.1)$ & $17(85.0)$ & $50(83.3)$ \\
\hline Moderate & $889(41.9)$ & $881(43.2)$ & $8(10.0)$ & $161(37.4)$ & $720(44.7)$ & $1(5.0)$ & $7(11.7)$ \\
\hline Hazardous & $353(16.6)$ & $348(17.1)$ & $5(6.3)$ & $40(9.3)$ & $308(19.1)$ & $2(10.0)$ & $3(5.0)$ \\
\hline \multicolumn{8}{|c|}{ Attitude to effect of drinking on AIDS } \\
\hline Good & $8(2.2)$ & $8(2.3)$ & $0(0.0)$ & $8(2.3)$ & - & $0(0.0)$ & - \\
\hline Harmful & $266(72.5)$ & 255 (72.2) & $11(78.6)$ & $255(72.2)$ & - & $11(78.6)$ & - \\
\hline No effect & $93(25.3)$ & $90(25.5)$ & $3(21.4)$ & $90(25.5)$ & - & $3(21.4)$ & - \\
\hline \multicolumn{8}{|c|}{ Ever tried to quit drinking after diagnosed with HIV } \\
\hline Yes & $181(49.3)$ & $174(49.3)$ & $7(50.0)$ & $174(49.3)$ & - & $7(50.0)$ & - \\
\hline No & $186(50.7)$ & 179 (50.7) & $7(50.0)$ & $179(50.7)$ & - & $7(50.0)$ & - \\
\hline
\end{tabular}

${ }^{* *} p<0.01 ;{ }^{* * *} p<0.001$

tp Value obtained from $\chi^{2}$ tests or Fisher's exact tests. 
Table 5 Prevalence and associated factors of alcohol drinking quantity among study participants

\begin{tabular}{|c|c|c|c|c|c|}
\hline Variables & $\begin{array}{l}\text { None } \\
(\mathrm{N}=879)\end{array}$ & $\begin{array}{l}\text { Moderate } \\
(\mathrm{N}=889)\end{array}$ & $\begin{array}{l}\text { Hazardous } \\
(\mathrm{N}=353)\end{array}$ & Crude OR (95\% Cl) & Adjusted OR (95\% Cl)† \\
\hline \multicolumn{6}{|l|}{ Gender } \\
\hline Female & $67(83.8)$ & $8(10.0)$ & $5(6.3)$ & 1.00 & 1.00 \\
\hline Male & $812(39.8)$ & $881(43.2)$ & $348(17.1)$ & $7.43(4.12 \text { to } 13.42)^{\star \star \star}$ & $7.20(3.83 \text { to } 13.54)^{\star \star \star}$ \\
\hline \multicolumn{6}{|l|}{ Age (years) } \\
\hline $18-29$ & 107 (42.6) & $114(45.4)$ & $30(12.0)$ & 1.00 & 1.00 \\
\hline 30-39 & 375 (46.2) & $338(41.7)$ & $98(12.1)$ & $0.90(0.69$ to 1.18$)$ & $1.22(0.91$ to 1.64$)$ \\
\hline $40-49$ & $258(39.5)$ & $272(41.7)$ & $123(18.8)$ & $1.26(0.96$ to 1.66$)$ & $1.70(1.23 \text { to } 2.35)^{\star \star}$ \\
\hline $50-79$ & 139 (34.2) & $165(40.6)$ & $102(25.1)$ & $1.69(1.26 \text { to } 2.28)^{\star \star}$ & $1.76(1.22 \text { to } 2.53)^{\star \star}$ \\
\hline \multicolumn{6}{|l|}{ Ethnicity } \\
\hline Han & 498 (56.3) & 297 (33.6) & $90(10.2)$ & 1.00 & 1.00 \\
\hline Jingpo & 267 (29.4) & $452(49.7)$ & 190 (20.9) & $2.85(2.38 \text { to } 3.42)^{* * *}$ & $2.58(2.11 \text { to } 3.16)^{\star \star *}$ \\
\hline Dai & 74 (29.7) & $118(47.4)$ & 57 (22.9) & $2.97(2.27 \text { to } 3.88)^{\star \star \star}$ & $3.08(2.31 \text { to } 4.11)^{\star \star \star}$ \\
\hline Others & $40(51.3)$ & 22 (28.2) & $16(20.5)$ & 1.45 (0.93 to 2.25$)$ & 1.56 (0.99 to 2.47$)$ \\
\hline \multicolumn{6}{|l|}{ Marital status } \\
\hline Never married & $184(48.7)$ & 147 (38.9) & $47(12.4)$ & 1.00 & 1.00 \\
\hline Currently married & 590 (39.5) & $639(42.7)$ & $266(17.8)$ & $1.47(1.19 \text { to } 1.82)^{\star \star}$ & $0.93(0.70$ to 1.24$)$ \\
\hline Divorced or widowed & 105 (42.3) & $103(41.5)$ & $40(16.1)$ & 1.31 (0.96 to 1.77$)$ & $1.17(0.85$ to 1.63$)$ \\
\hline \multicolumn{6}{|l|}{ Education level } \\
\hline Illiteracy or primary & $345(32.5)$ & $487(45.8)$ & $231(21.7)$ & 1.00 & 1.00 \\
\hline Middle & $398(48.8)$ & $318(39.0)$ & $100(12.3)$ & $0.50(0.42 \text { to } 0.60)^{\star \star *}$ & $0.76(0.63 \text { to } 0.93)^{\star \star}$ \\
\hline High or above & $136(56.2)$ & 84 (34.7) & $22(9.1)$ & $0.37(0.28 \text { to } 0.49)^{* \star *}$ & $0.65(0.48 \text { to } 0.89)^{\star \star}$ \\
\hline \multicolumn{6}{|c|}{ Age at first drug use (years) } \\
\hline$<25$ & $544(45.4)$ & $472(39.4)$ & $183(15.3)$ & 1.00 & 1.00 \\
\hline$\geq 25$ & 335 (36.3) & 417 (45.2) & $170(18.4)$ & $1.39(1.18 \text { to } 1.64)^{\star \star \star}$ & $0.89(0.73$ to 1.09$)$ \\
\hline \multicolumn{6}{|l|}{ Length of MMT (years) } \\
\hline$<1$ & $115(31.3)$ & $203(55.3)$ & $49(13.4)$ & 1.00 & 1.00 \\
\hline $1-5$ & 417 (38.4) & $484(44.6)$ & $184(17.0)$ & $0.89(0.71$ to 1.11$)$ & $0.92(0.73$ to 1.16$)$ \\
\hline$>5$ & 347 (51.9) & $202(30.2)$ & 120 (17.9) & $0.60(0.47 \text { to } 0.76)^{\star \star \star}$ & $0.77(0.59 \text { to } 1.00)^{*}$ \\
\hline \multicolumn{6}{|c|}{ Daily methadone dose (mL) } \\
\hline$\leq 30$ & $133(39.7)$ & $150(44.8)$ & $52(15.5)$ & 1.00 & 1.00 \\
\hline $31-60$ & $281(36.7)$ & 327 (42.7) & $157(20.5)$ & $1.17(0.93$ to 1.46$)$ & $1.29(1.02 \text { to } 1.63)^{*}$ \\
\hline $61-99$ & $228(43.2)$ & $217(41.1)$ & $83(15.7)$ & $0.87(0.68$ to 1.11$)$ & $1.04(0.81$ to 1.35$)$ \\
\hline$\geq 100$ & $213(51.8)$ & $146(35.5)$ & $52(12.7)$ & $0.63(0.48 \text { to } 0.81)^{\star *}$ & $0.97(0.73$ to 1.29$)$ \\
\hline \multicolumn{6}{|c|}{ Number of sexual partners in the last year } \\
\hline 0 & $286(47.6)$ & $226(37.6)$ & $89(14.8)$ & 1.00 & 1.00 \\
\hline 1 & $568(39.4)$ & $625(43.4)$ & $248(17.2)$ & $1.35(1.13 \text { to } 1.61)^{\star *}$ & $1.34(1.04 \text { to } 1.73)^{*}$ \\
\hline$\geq 2$ & $23(30.7)$ & $36(48.0)$ & $16(21.3)$ & $1.88(1.20 \text { to } 2.94)^{\star *}$ & $2.46(1.53 \text { to } 3.97)^{\star *}$ \\
\hline \multicolumn{6}{|c|}{ Smoking intensity in the past month } \\
\hline Low & $129(57.1)$ & $63(27.9)$ & $34(15.0)$ & 1.00 & 1.00 \\
\hline Moderate & $203(41.5)$ & $227(46.4)$ & $59(12.1)$ & $1.56(1.15 \text { to } 2.11)^{\star \star}$ & $1.11(0.80$ to 1.55$)$ \\
\hline High & 547 (38.9) & $599(42.6)$ & $260(18.5)$ & $1.92(1.46 \text { to } 2.52)^{\star \star \star}$ & $1.39(1.03 \text { to } 1.88)^{*}$ \\
\hline \multicolumn{6}{|l|}{ HIV infection } \\
\hline No & $632(37.8)$ & $727(43.5)$ & $311(18.6)$ & 1.00 & 1.00 \\
\hline Yes & $247(54.8)$ & $162(35.9)$ & $42(9.3)$ & $0.49(0.40 \text { to } 0.60)^{\star \star *}$ & $0.53(0.42 \text { to } 0.68)^{* * *}$ \\
\hline \multicolumn{6}{|l|}{ HCV infection } \\
\hline No & $504(37.8)$ & $598(44.8)$ & $233(17.5)$ & 1.00 & 1.00 \\
\hline Yes & $375(47.7)$ & $291(37.0)$ & $120(15.3)$ & $0.71(0.60 \text { to } 0.84)^{* * *}$ & $0.86(0.71$ to 1.04$)$ \\
\hline
\end{tabular}

${ }^{*} p<0.05 ;{ }^{* *} p<0.01 ;{ }^{* * *} p<0.001$.

†Adjusted for potential confounding variables listed in the table by multivariate ordinal logistic regression analysis.

with marital status, age of first drug use, daily methadone dose or HCV infection.

\section{DISCUSSION}

In this cross-sectional study with 2121 MMT participants from western China, we observed the overall prevalence of ever, current and heavy/hazardous for smoking was $98.6 \%, 97.8 \%$ and $66.3 \%$, respectively; and the prevalence for alcohol drinking was $86.6 \%, 58.6 \%$ and $16.6 \%$, respectively. We also found significantly different patterns of tobacco and alcohol consumption when comparing men and women, or HIV-positive and negative participants. Moreover, we reported that gender, age, 
ethnicity, level of education and sexual activity were associated with smoking and drinking behaviours, while length of MMT and HIV infection were negatively associated with heavier drinking. Smoking and drinking were also correlated with each other in the models.

MMT patients in the survey showed an overall prevalence of current smoking of $97.8 \%$, which was more than three times that of $28.3 \%$ in Chinese general adults. When looking by gender, the prevalence of current smoking was about twice and 30 times than that of Chinese men and women in the general population, respectively (men: $98.8 \%$ vs $53.3 \%$, women: $73.7 \%$ vs $2.5 \%) .{ }^{42}$ Among MMT patients in western counties, the overall prevalence of smoking was observed from $78.5 \%$ to $93.0 \% ;{ }^{17-20}$ while there have been only two previous studies reported current smoking prevalence (91.4\% and $92.9 \%$, respectively) among Chinese MMT patients. ${ }^{23}{ }^{24}$ Previous studies' sample sizes were $<600$, but consistent with our results, high prevalence of smoking was uniformly observed among MMT patients.

To the best of our knowledge, no previous study had reported the prevalence of heavy smoking among MMT patients in China. There was only one study measuring heavy smoking using the same method as ours; however, it only recruited 32 MMT participants from Los Angeles and reported 11 heavy smokers. ${ }^{43}$ Of all participants in our analysis, the prevalence of overall heavy smoking was $66.3 \%, 68.1 \%$ men and $20.0 \%$ women, which were extremely high compared with the general Chinese population aged 15 years and above (overall: $10.1 \%$, men: $22.8 \%$ and women: $0.6 \%),{ }^{44}$ and were higher than those of $2.6 \%$ and $7.2 \%$ of adults in California and the remaining USA in 2007, respectively. ${ }^{39}$ As almost all MMT patients are smokers, smoking intensity may be a better exposure measure when examining the seriousness of smoking effects on health. Meanwhile, the published literature suggested that interventions among MMT patients have been largely unsuccessful in achieving sustained smoking abstinence. ${ }^{45}$ Therefore, implementation of innovative smoking cessation programmes is urgent and smoking reduction may be the primary and more important intervention among heavy smokers.

The overall prevalence of current alcohol drinking in the study population was $58.6 \%$, which was twice that of Chinese general adults $(28.8 \%){ }^{46}$ This prevalence was higher than that $(30.3 \%)$ in central China, ${ }^{32}$ and also higher than that $(44.0 \%$ and $49.0 \%)$ among MMT patients of the Beth Israel Medical Center (BIMC) in the USA. ${ }^{26} 27$ The prevalence $(16.6 \%)$ of participating in hazardous alcohol drinking among all study participants was lower than those (22-35\%) reported in BIMC with the same criterion of hazardous alcohol level. ${ }^{25-27}$ We observed that the proportion declined by $28.0 \%$ from ever drinking to current drinking, especially for women (by 50.1\%), suggesting that alcohol consumption was negatively affected during the course of MMT, although a systematic review found no change when patients had been on MMT (three studies supported an increase, three supported a decrease and nine supported no change in alcohol use). ${ }^{29}$

It was not surprising to find that heavier smoking and higher level alcohol drinking in the preceding month were positively associated with men, which was observed in the Chinese general population. ${ }^{47}$ Meanwhile, results showed that those who were older and had little education were more likely to be heavier smokers and hazardous drinkers, which was not consistent with previous results. ${ }^{31}$ Ethnic minorities were more likely than ethnic Han to engage in high level of alcohol consumption, which was consistent with the finding among HIV-infected patients who were also from Dehong Prefecture. ${ }^{37}$ But ethnic minorities showed less heavier smoking, which might be due to different ethnic cultures. ${ }^{49}$ This suggested that tobacco and alcohol cessation programmes in future should be mindfully conducted according to different demographic characteristics in target populations.

As mentioned above, patients in MMT were more likely to drink less, and hazardous drinking was further found to be negatively associated with the length of receiving MMT. In addition, of PLWHA in our study, a large proportion $(72.5 \%)$ have realised the harmful effects of alcohol on AIDS, and half of them ever tried to quit drinking. Therefore, it was not surprising that hazardous drinking was observed to be negatively associated with HIV infection, whereas no difference of ever drinking was observed between HIV-infected individuals and HIV-uninfected individuals. Meanwhile, a relatively small percentage were aware of tobacco's harmful effects and less than a quarter ever tried to quit smoking, resulting in a null association between heavier smoking and HIV infection. It will be of great value to enhance MMT patients' awareness of harmful effects of tobacco and alcohol consumption by carrying out health education programmes.

Furthermore, risky behaviours of heavier smoking and more hazardous drinking were positively associated with each other, and both were positively associated with more sexual partners in this study. This might probably explain or be due to the fact that DUs worldwide are likely to engage in high levels of risky sexual behaviours $^{50} 51$ and often have coexisted with illegal substance use behaviours. ${ }^{52}$ Consistent with our results, previous studies have shown that alcohol drinking was positively associated with a number of sexual risk behaviours including multiple sexual partners. ${ }^{25} 2753$

\section{Limitations}

This study has certain limitations. First, similar to all cross-sectional studies, causal inferences cannot be made. Rather, we are reporting the subgroups of participants with even higher prevalence of use to target the population who are at higher risk for alcohol and tobacco-related health problems for focused intervention. Second, self-reported data were used for health-related risk behaviours and behaviours in the 
past. There might be under-reporting of health-related risk behaviours such as number of sexual partners in the last year. And it could be more difficult to recall the memory after a long time on details such as age at first drug use, smoking/drinking initiation age, etc. So information bias may exist in our study. However, the primary variables asked about in the preceding month prior to interviews were asked by well-trained public health professional in a private setting that will minimise recall bias and deliberate concealment of sensitive personal topics. Third, the sample size of female MMT patients in the study was only $80(3.8 \%)$, and substantial differences were not found significant when comparing by HIV status in women. A study with larger sample size of women is needed to better explore the association between characteristics and behaviours with alcohol and tobacco use in female DUs receiving MMT. Fourth, all participants were recruited from Dehong, the observed results of tobacco smoking and alcohol drinking in our study might not be generalisable to MMT patients in other areas, and may not be generalisable to those DUs who do not attend MMT clinic. Fifth, the validity and reliability of questions measuring tobacco and alcohol use should be specifically verified among MMT patients in future research, although these questions were previously used for HIV-infected people including HIV-infected DUs in the same study area. ${ }^{37} 38$

\section{CONCLUSIONS}

The present study suggested high prevalence of current tobacco smoking, current alcohol drinking, heavy smoking and hazardous drinking among MMT patients in China. It is vital to implement comprehensive education and effective intervention programmes to reduce the harmful use of tobacco and alcohol among MMT patients. Furthermore, the comparative risk assessment of disease burden attributable to tobacco and alcohol consumption, and the evidence for the effectiveness and cost-effectiveness of interventions to prevent and reduce tobacco and alcoholrelated harm are needed in the further studies. In addition, confirmation of associated factors, changing trends in the prevalence and comorbid mental health conditions of tobacco and alcohol use among MMT patients warrant further longitudinal cohort studies.

\section{Author affiliations \\ ${ }^{1}$ Dehong Prefecture Center for Disease Control and Prevention, Mangshi, Yunnan Province, China \\ ${ }^{2}$ Department of Epidemiology at School of Public Health and The Key Laboratory of Public Health Safety of Ministry of Education, Fudan University, Shanghai, China \\ ${ }^{3}$ Collaborative Innovation Center of Social Risks Governance in Health, Fudan University, Shanghai, China}

Acknowledgements The authors are grateful to Dr Aileen Baecker from UCLA School of Public Health for her valuable comments and editorial assistance.

Contributors The study was conceived and supervised by NH and SD. YY, $R Y, R T, M G$ and YD conducted the survey. ZJ analysed the data and drafted the manuscript. $\mathrm{XL}$ and $\mathrm{NH}$ critically reviewed and revised the manuscript.
Funding This work was supported by National Natural Science Foundation of China (grant numbers 81373062, 81161120407 and 81361120385), Shanghai Municipal Bureau of Health (grant number GWTD2015S05) and China National Major AIDS Research Project (2012ZX10001001-004).

Competing interests None declared.

Patient consent Obtained.

Ethics approval The Institutional Review Board (IRB) of Fudan University.

Provenance and peer review Not commissioned; externally peer reviewed.

Data sharing statement No additional data are available.

Open Access This is an Open Access article distributed in accordance with the Creative Commons Attribution Non Commercial (CC BY-NC 4.0) license, which permits others to distribute, remix, adapt, build upon this work noncommercially, and license their derivative works on different terms, provided the original work is properly cited and the use is non-commercial. See: http:// creativecommons.org/licenses/by-nc/4.0/

\section{REFERENCES}

1. Pang L, Hao Y, Mi G, et al. Effectiveness of first eight methadone maintenance treatment clinics in China. AIDS 2007;21(Suppl 8): S103-7.

2. Yin $W$, Hao $Y$, Sun $X$, et al. Scaling up the national methadone maintenance treatment program in China: achievements and challenges. Int J Epidemiol 2010;39(Suppl 2):ii29-37.

3. $\mathrm{Li} \mathrm{J}, \mathrm{Li} \mathrm{X}$. Current status of drug use and HIV/AIDS prevention in drug users in China. J Food Drug Anal 2013;21:S37-41.

4. Sun HM, Li XY, Chow EP, et al. Methadone maintenance treatment programme reduces criminal activity and improves social well-being of drug users in China: a systematic review and meta-analysis. BMJ Open 2015;5:e005997.

5. Fareed A, Casarella J, Amar R, et al. Benefits of retention in methadone maintenance and chronic medical conditions as risk factors for premature death among older heroin addicts. J Psychiatr Pract 2009;15:227-34.

6. Loughlin AM, Schwartz R, Strathdee SA. Prevalence and correlates of $\mathrm{HCV}$ infection among methadone maintenance attendees: implications for HCV treatment. Int J Drug Policy 2004;15:93-101.

7. Zhuang X, Liang Y, Chow EP, et al. HIV and HCV prevalence among entrants to methadone maintenance treatment clinics in China: a systematic review and meta-analysis. BMC Infect Dis 2012;12:130.

8. Helleberg M, Afzal S, Kronborg G, et al. Mortality attributable to smoking among HIV-1-infected individuals: a nationwide, population-based cohort study. Clin Infect Dis 2013;56:727-34.

9. Samet JH, Cheng DM, Libman $\mathrm{H}$, et al. Alcohol consumption and HIV disease progression. J Acquir Immune Defic Syndr 2007;46:194-9.

10. Cioe PA, Baker J, Kojic EM, et al. Elevated soluble CD14 and lower D-Dimer are associated with cigarette smoking and heavy episodic alcohol use in persons living with HIV. J Acquir Immune Defic Syndr 2015;70:400-5.

11. Zhang J, Ou JX, Bai CX. Tobacco smoking in China: prevalence, disease burden, challenges and future strategies. Respirology 2011;16:1165-72

12. World Health Organization. Global status report on alcohol and health 2011. http://www.who.int/substance_abuse/publications/ global_alcohol_report/msbgsruprofiles.pdf

13. Lopez AD, Mathers CD, Ezzati M, et al. Global and regional burden of disease and risk factors, 2001: systematic analysis of population health data. Lancet 2006;367:1747-57.

14. Gu D, Kelly TN, Wu X, et al. Mortality attributable to smoking in China. N Engl J Med 2009;360:150-9.

15. Secretan B, Straif K, Baan R, et al. A review of human carcinogensPart E: tobacco, areca nut, alcohol, coal smoke, and salted fish Lancet Oncol 2009;10:1033-4.

16. Park LS, Hernandez-Ramirez RU, Silverberg MJ, et al. Prevalence of non-HIV cancer risk factors in persons living with HIV/AIDS: a meta-analysis. AIDS 2016;30:273-91.

17. Best $\mathrm{D}$, Lehmann $\mathrm{P}$, Gossop $\mathrm{M}$, et al. Eating too little, smoking and drinking too much: wider lifestyle problems among methadone maintenance patients. Addict Res Theory 1998;6:489-98.

18. Clarke JG, Stein MD, McGarry KA, et al. Interest in smoking cessation among injection drug users. Am J Addict 2001;10:159-66.

19. Richter KP, Gibson CA, Ahluwalia JS, et al. Tobacco use and quit attempts among methadone maintenance clients. Am J Public Health 2001;91:296-9. 
20. Stark MJ, Campbell BK. Drug use and cigarette smoking in applicants for drug abuse treatment. J Subst Abuse 1993;5:175-81.

21. Du WJ, Xiang YT, Wang ZM, et al. Socio-demographic and clinical characteristics of 3129 heroin users in the first methadone maintenance treatment clinic in China. Drug Alcohol Depend 2008;94:158-64.

22. Li X, Zhou Y, Stanton B. Illicit drug initiation among institutionalized drug users in China. Addiction 2002;97:575-82.

23. Zhu J, Zhong B, L Y. Cigarette smoking behavior and influencing factors among methadone maintenance treatment outpatients. Chin $J$ Public Health 2012;28:673-6.

24. Li L, Zhou W. Relationship between the smoking rate and heroin abuse in male and female heroin addicts. Chin J Drug Abuse Prev Treat 2008;14:265-7.

25. Arasteh K, Des Jarlais DC. At-risk drinking and injection and sexual risk behaviors of HIV-positive injection drug users entering drug treatment in New York City. AIDS Patient Care STDS 2009;23:657-61.

26. Arasteh K, Des Jarlais DC. HIV testing and treatment among at-risk drinking injection drug users. J Int Assoc Physicians AIDS Care (Chic) 2009;8:196-201.

27. Arasteh K, Des Jarlais DC, Perlis TE. Alcohol and HIV sexual risk behaviors among injection drug users. Drug Alcohol Depend 2008;95:54-61.

28. Rengade CE, Kahn JP, Schwan R. Misuse of alcohol among methadone patients. Am J Addict 2009;18:162-6.

29. Srivastava A, Kahan M, Ross S. The effect of methadone maintenance treatment on alcohol consumption: a systematic review. J Subst Abuse Treat 2008:34:215-23.

30. Du J, Wang Z, Xie B, et al. Hepatitis C knowledge and alcohol consumption among patients receiving methadone maintenance treatment in Shanghai, China. Am J Drug Alcohol Abuse 2012;38:228-32.

31. Chen IC, Chie WC, Hwu HG, et al. Alcohol use problem among patients in methadone maintenance treatment in Taiwan. $J$ Subst Abuse Treat 2011;40:142-9.

32. Wang J, Zhong B, Zhu J, et al. Alcohol drinking behavior among methadone maintenance treatment outpatients: characteristics and related factors. Med J Chin People's Health 2013;25:30-3.

33. Liu X, Pan S, Zhang X. Alcohol abuse on the patients with methadone maintain treatment. Chin J Drug Abuse Prev Treat 2008;14:205-6.

34. Wu Z, Sullivan SG, Wang Y, et al. Evolution of China's response to HIV/AIDS. Lancet 2007;369:679-90.

35. Jia M, Luo H, Ma Y, et al. The HIV epidemic in Yunnan Province, China, 1989-2007. J Acquir Immune Defic Syndr 2010;53(Suppl 1): S34-40.

36. Wang R, Ding $\mathrm{Y}$, Bai $\mathrm{H}$, et al. Illicit heroin and methamphetamine use among methadone maintenance treatment patients in Dehong Prefecture of Yunnan Province, China. PLoS ONE 2015;10: e0133431.
37. Luo X, Duan S, Duan Q, et al. Alcohol use and subsequent sex among HIV-infected patients in an ethnic minority area of Yunnan Province, China. PLoS ONE 2013;8:e61660.

38. Luo X, Duan S, Duan Q, et al. Tobacco use among HIV-infected individuals in a rural community in Yunnan Province, China. Drug Alcohol Depend 2014;134:144-50.

39. Pierce JP, Messer K, White MM, et al. Prevalence of heavy smoking in California and the United States, 1965-2007. JAMA 2011;305:1106-12.

40. Lu S, Du S, Ma G. The progress of standard alcohol drink measurement. J Hyg Res 2015;44:163-6.

41. National Institute on Alcohol Abuse and Alcoholism. Helping patients who drink too much: a clinician's guide, Updated 2005 edn. 2005. https://pubs.niaaa.nih.gov/publications/Practitioner/ CliniciansGuide2005/guide.pdf

42. Zhang M, Wang LM, Li YC, et al. Cross-sectional survey on smoking and smoking cessation behaviors among Chinese adults in 2010. Zhonghua Yu Fang Yi Xue Za Zhi 2012;46:404-8.

43. Frosch DL, Shoptaw S, Nahom D, et al. Associations between tobacco smoking and illicit drug use among methadone-maintained opiate-dependent individuals. Exp Clin Psychopharmacol 2000;8:97-103.

44. Ma G, Kong L, Luan D, et al. The descriptive analysis of the smoking pattern of People in China. Chin J Prev Contr Chron Non-commun Dis 2005;13:195-9.

45. Okoli CT, Khara M, Procyshyn RM, et al. Smoking cessation interventions among individuals in methadone maintenance: a brief review. J Subst Abuse Treat 2010;38:191-9.

46. Ma G, Zhu D, Hu X, et al. The drinking practice of people in China Acta Nutr Sin 2005;27:362-5.

47. Yang T, Barnett R, Jiang S, et al. Gender balance and its impact on male and female smoking rates in Chinese cities. Soc Sci Med 2016;154:9-17.

48. Hao W, Su Z, Liu B, et al. Drinking and drinking patterns and health status in the general population of five areas of China. Alcohol Alcohol 2004;39:43-52.

49. Li J, Zhao X, Li Z, et al. Alcohol use and social changes among ethnic minorities in Yunnan, China. Shanghai Arch Psychiatry 2010;22:440-3.

50. Abdala N, Krasnoselskikh TV, Durante AJ, et al. Sexually transmitted infections, sexual risk behaviors and the risk of heterosexual spread of HIV among and beyond IDUs in St. Petersburg, Russia. Eur Addict Res 2008;14:19-25.

51. Yao Y, Wang N, Chu J, et al. Sexual behavior and risks for HIV infection and transmission among male injecting drug users in Yunnan, China. Int J Infect Dis 2009;13:154-61.

52. Benard A, Bonnet F, Tessier JF, et al. Tobacco addiction and HIV infection: toward the implementation of cessation programs. ANRS CO3 Aquitaine Cohort. AIDS Patient Care STDS 2007:21:458-68.

53. Yang $X$, Latkin $C$, Celentano D, et al. Prevalence and correlates of HIV risk behaviors among drug users in China. AIDS Behav 2006;10:71-81. 